STRUCTURE REPORTS

ISSN 1600-5368

Received 22 April 2014

Accepted 16 May 2014

Edited by W. T. A. Harrison, University of Aberdeen, Scotland

This work forms part of the PhD thesis (Neuchâtel, 1999) of TA.

Keywords: crystal structure; tetrakis-substituted; pyrazine; chiral

CCDC reference: 1004263

Supporting information: this article has supporting information at journals.iucr.org/e

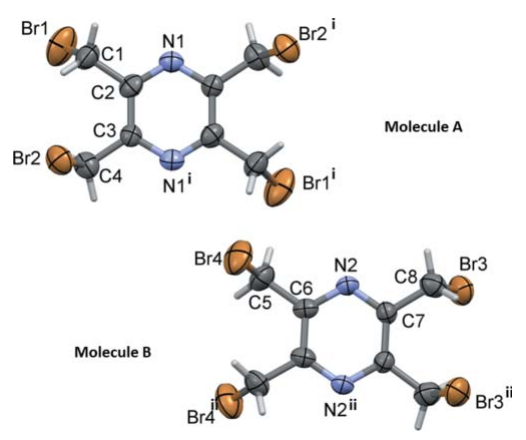

OPEN $\odot$ ACCESS

\section{Crystal structure of a tetrakis-substituted pyrazine compound: 2,3,5,6-tetrakis(bromomethyl)pyrazine}

\author{
Tokouré Assoumatine ${ }^{\mathrm{a}}$ and Helen Stoeckli-Evans ${ }^{\mathrm{b} *}$
}

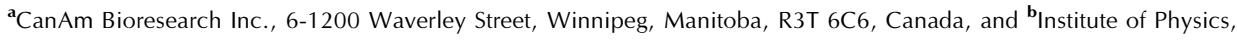
University of Neuchâtel, rue Emile-Argand 11, CH-2000 Neuchâtel, Switzerland. *Correspondence e-mail: helen.stoeckli-evans@unine.ch

The title compound, $\mathrm{C}_{8} \mathrm{H}_{8} \mathrm{Br}_{4} \mathrm{~N}_{2}$, crystallizes in the enantiomorphic-defining space group $P 4_{1} 2_{1} 2$ and has a refined Flack $x$ parameter of 0.04 (4). In the asymmetric unit, there are two half-molecules; the whole molecules ( $A$ and $B$ ) are generated by twofold rotation symmetry. In molecule $A$, the twofold axis is normal to the pyrazine ring passing through the centre of the ring, while in molecule $B$, the twofold rotation axis lies in the plane of the pyrazine ring bisecting the $\mathrm{C}-\mathrm{C}$ aromatic bonds. The two molecules are pseudo-mirror images of one another, and the best fit of the two molecules was obtained for inverted molecule $B$ on molecule $A$, with an r.m.s. deviation of $0.1048 \AA$ and a maximum deviation of any two equivalent atoms of $0.2246 \AA$. In the crystal, the $A$ molecules are linked by weak $\mathrm{C}-\mathrm{H} \cdots \mathrm{Br}$ hydrogen bonds and $\mathrm{Br} \cdots \mathrm{Br}$ interactions [3.524 (3) $\AA$ ], forming a three-dimensional framework. The $B$ molecules are also linked by weak $\mathrm{C}-\mathrm{H} \cdots \mathrm{Br}$ hydrogen bonds and $\mathrm{Br} \cdots \mathrm{Br}$ interactions [3.548 (3) $\mathrm{A}]$, forming a three-dimensional network that interpenetrates the network of $A$ molecules.

\section{Chemical context}

The title compound is the starting material used for the synthesis of a number of 2,3,5,6-tetrakis-substituted pyrazine compounds (Ferigo et al., 1994; Assoumatine, 1999). For example, 2,3,5,6-tetrakis(aminomethyl)pyrazine has been used as a ligand to prepare copper(II), zinc(II) and manganese(II) binuclear and polymeric complexes (Ferigo et al., 1994).<smiles>BrCc1nc(CBr)c(CBr)nc1CBr</smiles>

\section{Structural commentary}

The title compound, Fig. 1, crystallizes with two half-molecules per asymmetric unit. The whole molecules $(A$ and $B)$ are generated by twofold rotation symmetry. In molecule $A$, the twofold axis is normal to the pyrazine ring passing through the centre of the ring. In molecule $B$, the twofold rotation axis lies in the plane of the pyrazine ring bisecting the $\mathrm{C} 6-6^{\mathrm{ii}}$ and $\mathrm{C} 7-$ $\mathrm{C} 7^{\mathrm{ii}}$ bonds [symmetry code: (ii) $y, x,-z$ ]. Placed side by side, it can be seen that the two molecules are almost perfect mirror images of each other (Fig. 1). The best fit of the two molecules, 
Table 1

Selected torsion angles $\left({ }^{\circ}\right)$.

\begin{tabular}{lrlr}
\hline $\mathrm{Br} 1-\mathrm{C} 1-\mathrm{C} 2-\mathrm{N} 1$ & $91.3(13)$ & $\mathrm{Br} 4-\mathrm{C} 5-\mathrm{C} 6-\mathrm{N} 2$ & $-93.3(12)$ \\
$\mathrm{Br} 1-\mathrm{C} 1-\mathrm{C} 2-\mathrm{C} 3$ & $-92.6(15)$ & $\mathrm{Br} 4-\mathrm{C} 5-\mathrm{C} 6-\mathrm{C6}^{\mathrm{ii}}$ & $84.8(17)$ \\
$\mathrm{N} 1{ }^{\mathrm{i}}-\mathrm{C} 3-\mathrm{C} 4-\mathrm{Br} 2$ & $103.1(11)$ & $\mathrm{N} 2-\mathrm{C} 7-\mathrm{C} 8-\mathrm{Br} 3$ & $-101.0(12)$ \\
$\mathrm{C} 2-\mathrm{C} 3-\mathrm{C} 4-\mathrm{Br} 2$ & $-78.6(15)$ & $\mathrm{C} 7^{\mathrm{i}}-\mathrm{C} 7-\mathrm{C} 8-\mathrm{Br} 3$ & $77.4(18)$ \\
\hline
\end{tabular}

Symmetry codes: (i) $-y+1,-x+1,-z+\frac{1}{2}$; (ii) $y, x,-z$.

Table 2

Hydrogen-bond geometry $\left(\AA,^{\circ}\right)$.

\begin{tabular}{lllll}
\hline$D-\mathrm{H} \cdots A$ & $D-\mathrm{H}$ & $\mathrm{H} \cdots A$ & $D \cdots A$ & $D-\mathrm{H} \cdots A$ \\
\hline $\mathrm{C} 1-\mathrm{H} 1 A \cdots \mathrm{Br} 2^{\mathrm{iii}}$ & 0.97 & 3.02 & $3.863(14)$ & 146 \\
$\mathrm{C} 1-\mathrm{H} 1 B \cdots \mathrm{Br} 2$ & 0.97 & 2.86 & $3.617(16)$ & 135 \\
$\mathrm{C} 5-\mathrm{H} 5 A \cdots \mathrm{Br} 4^{\mathrm{ii}}$ & 0.97 & 3.04 & $3.748(16)$ & 131 \\
$\mathrm{C} 5-\mathrm{H} 5 B \cdots \mathrm{Br} 3^{\text {iv }}$ & 0.97 & 3.03 & $3.864(14)$ & 145 \\
$\mathrm{C} 8-\mathrm{H} 8 A \cdots \mathrm{Br} 3^{\mathrm{ii}}$ & 0.97 & 2.96 & $3.654(15)$ & 130
\end{tabular}

Symmetry codes: (ii) $y, x,-z$; (iii) $-y+\frac{3}{2}, x-\frac{1}{2}, z+\frac{1}{4}$; (iv) $-x+\frac{1}{2}, y+\frac{1}{2},-z+\frac{1}{4}$.

calculated using the Molecular Overlay routine in Mercury (Macrae et al., 2008), was obtained for inverted molecule $B$ on molecule $A$ with an r.m.s. deviation of $0.1048 \AA$ and a maximum deviation of any two equivalent atoms of $0.2246 \AA$.

The main difference appears for the torsion angles $\mathrm{Br} 1-$ $\mathrm{C} 1-\mathrm{C} 2-\mathrm{C} 3=-92.6(15)^{\circ}$ in molecule $A$ and $\mathrm{Br} 4-\mathrm{C} 5-$ $\mathrm{C} 6-\mathrm{C}^{\mathrm{ii}}=84.8(17)^{\circ}$ in molecule $B$ [Table 1 ; symmetry code: (ii) $y, x,-z]$. The other torsion angles involving the $\mathrm{Br}-\mathrm{C}-$ $\mathrm{C}_{\mathrm{ar}}-\mathrm{C}_{\mathrm{ar}}(\mathrm{ar}=$ aromatic $)$ arms do not differ significantly (Table 2).

\section{Supramolecular features}

In the crystal, there are two interpenetrating three-dimensional networks composed of a network of $A$ molecules, linked by weak $\mathrm{C}-\mathrm{H} \cdots \mathrm{Br}$ hydrogen bonds and $\mathrm{Br} 1 \cdots \mathrm{Br} 2^{\mathrm{iii}}$ interactions [3.524 (3) $\AA$; symmetry code: (iii) $-y+2,-x+1$, $\left.-z+\frac{1}{2}\right]$, and a network of $B$ molecules, are also linked by weak $\mathrm{C}-\mathrm{H} \cdots \mathrm{Br}$ hydrogen bonds and $\mathrm{Br} 3 \cdots \mathrm{Br} 4^{\text {iv }}$ interactions
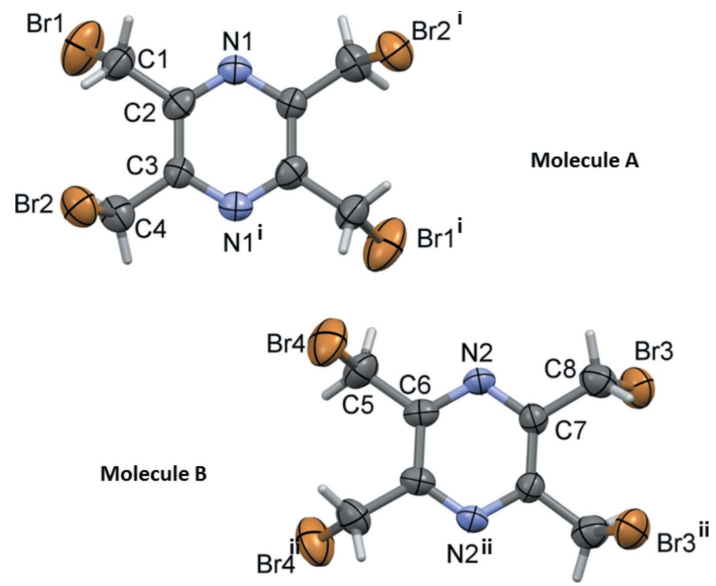

Figure 1

A view of the molecular structure of the two independent molecules ( $A$ and $B$ ) of the title compound, with atom labelling [symmetry codes: (i) $-y+1,-x+1,-z+\frac{1}{2}$; (ii) $\left.y, x,-z\right]$. The displacement ellipsoids are drawn at the $50 \%$ probability level.

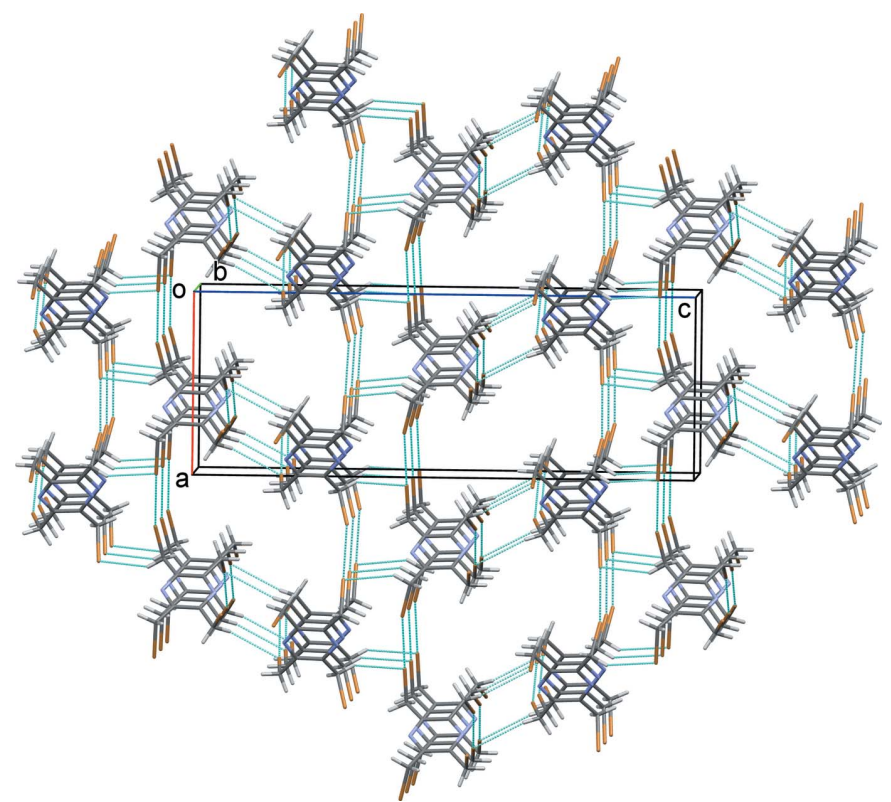

Figure 2

A view along the $b$ axis of the crystal packing of the $A$ molecules of the title compound. The weak $\mathrm{C}-\mathrm{H} \cdots \mathrm{Br}$ hydrogen bonds and $\mathrm{Br} \cdots \mathrm{Br}$ interactions are shown as dashed lines (see Table 2 for details).

[3.548 (3) ^, symmetry code: (iv) $x, y-1, z]$ (Table 2 and Fig. 3).

\section{Database survey}

A search of the Cambridge Structural Database (Version 5.33, last update November 2013; Allen, 2002) indicated the presence of a large number of tetrasubstituted pyrazine derivatives and their metal complexes, mainly involving

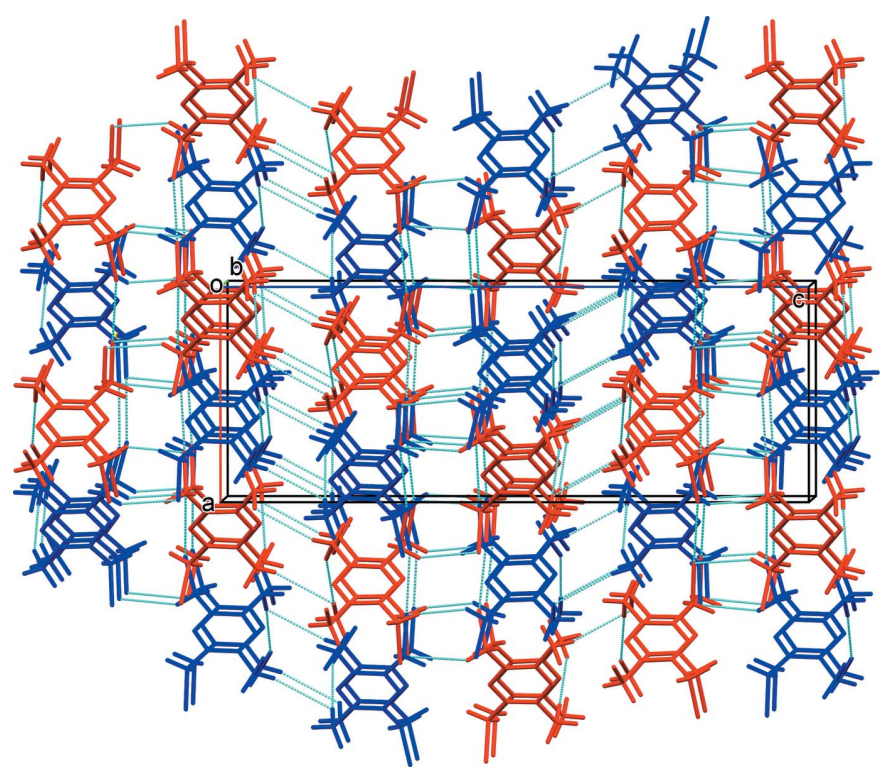

Figure 3

A view along the $b$ axis of the crystal packing of the title compound. The $\mathrm{C}-\mathrm{H} \cdots \mathrm{Br}$ hydrogen bonds and $\mathrm{Br} \cdots \mathrm{Br}$ interactions are shown as dashed lines (see Table 2 for details; $A$ molecules blue, $B$ molecules red). 
Table 3

Experimental details.

\begin{tabular}{|c|c|}
\hline \multicolumn{2}{|l|}{ Crystal data } \\
\hline Chemical formula & $\mathrm{C}_{8} \mathrm{H}_{8} \mathrm{Br}_{4} \mathrm{~N}_{2}$ \\
\hline$M_{\mathrm{r}}$ & 451.80 \\
\hline Crystal system, space group & Tetragonal, $P 4_{1} 2{ }_{1} 2$ \\
\hline Temperature $(\mathrm{K})$ & 293 \\
\hline$a, c(\AA)$ & $9.6858(4), 26.5116(17)$ \\
\hline$V\left(\AA^{3}\right)$ & $2487.2(3)$ \\
\hline$Z$ & 8 \\
\hline Radiation type & Мо $K \alpha$ \\
\hline$\mu\left(\mathrm{mm}^{-1}\right)$ & 12.91 \\
\hline Crystal size (mm) & $0.50 \times 0.40 \times 0.30$ \\
\hline \multicolumn{2}{|l|}{ Data collection } \\
\hline Diffractometer & Stoe IPDS 1 \\
\hline Absorption correction & $\begin{array}{l}\text { Multi-scan (MULscanABS in } \\
\quad \text { PLATON; Spek, 2009) }\end{array}$ \\
\hline$T_{\min }, T_{\max }$ & $0.430,1.000$ \\
\hline $\begin{array}{l}\text { No. of measured, independent and } \\
\text { observed }[I>2 \sigma(I)] \text { reflections }\end{array}$ & $19463,2417,1276$ \\
\hline$R_{\text {int }}$ & 0.113 \\
\hline$(\sin \theta / \lambda)_{\max }\left(\AA^{-1}\right)$ & 0.616 \\
\hline \multicolumn{2}{|l|}{ Refinement } \\
\hline$R\left[F^{2}>2 \sigma\left(F^{2}\right)\right], w R\left(F^{2}\right), S$ & $0.043,0.096,0.84$ \\
\hline No. of reflections & 2417 \\
\hline No. of parameters & 127 \\
\hline $\mathrm{H}$-atom treatment & $\mathrm{H}$-atom parameters constrained \\
\hline$\Delta \rho_{\max }, \Delta \rho_{\min }\left(\mathrm{e} \AA^{-3}\right)$ & $0.69,-0.54$ \\
\hline Absolute structure & $\begin{array}{l}\text { Flack } x \text { determined using } 419 \\
\left.\text { quotients }\left[\left(\mathrm{I}^{+}\right)-\mathrm{I}^{-}\right)\right] /\left[\left(\mathrm{I}^{+}\right)+\left(\mathrm{I}^{-}\right)\right] \\
(\text {Parsons \& Flack, 2004) }\end{array}$ \\
\hline Absolute structure parameter & $0.04(4)$ \\
\hline
\end{tabular}

Computer programs: EXPOSE, CELL and INTEGRATE in IPDS-I (Stoe \& Cie, 2004), SHELXS97 and SHELXL2013 (Sheldrick, 2008), Mercury (Macrae et al., 2008), PLATON (Spek, 2009) and publCIF (Westrip, 2010).

tetramethylpyrazine. A small number of them involve 2,3,5,6tetrakis(aminomethyl)pyrazine (tampyz), which was used to prepare transition metal binuclear complexes, for example $\left[\mathrm{Cl}_{2} \mathrm{Zn}\right.$ (tampyz) $\left.\mathrm{ZnCl}_{2}\right]$, and a quasi-linear one-dimensional coordination polymer, $\left\{\mathrm{Mn}\left(\text { tampyz) } \mathrm{Cl}_{2} \cdot 2 \mathrm{H}_{2} \mathrm{O}\right\}_{n}\right.$ (Ferigo et al., 1994). The title compound has also been used in the synthesis of two triclinic polymorphs of 2,3,5,6 tetrakis(naphthalen-2ylsulfanylmethyl)pyrazine (Pacifico \& Stoeckli-Evans, 2004), 2,3,5,6-tetrakis((naphthalen-2-yloxy)methyl)pyrazine (Gasser \& Stoeckli-Evans, 2007), 2,3,5,6-tetrakis(phenoxymethyl)pyrazine and 2,3,5,6-tetrakis(phenylsulfanylmethyl)pyrazine (Assoumatine et al., 2007). All five structures possess inversion symmetry. The sulfanyl derivatives crystallize in the triclinic space group $P \overline{1}$, while the oxy derivatives crystallize in the monoclinic space group $P 2_{1} / c$.

\section{Synthesis and crystallization}

The title compound was prepared by a modification of the procedure described by Ferigo et al. (1994). To 2,3,5,6-tetramethylpyrazine $(28 \mathrm{~g}, 0.28 \mathrm{~mol})$ in $\mathrm{CCl}_{4}(1 \mathrm{l})$ was added wellground $N$-bromosuccinimide $(150 \mathrm{~g}, 0.84 \mathrm{~mol})$. The mixture was stirred vigorously and heated to reflux. As soon as the reflux set in, the mixture was irradiated for $5 \mathrm{~h}$ with two $200 \mathrm{~W}$ lamps fitted at least $10 \mathrm{~cm}$ at opposite sides of the flask. After the mixture was then cooled firstly to room temperature and the floating succinimide filtered off. The orange filtrate was cooled overnight to $278 \mathrm{~K}$ to crystallize the remaining traces of succinimide, which was filtered off. The filtrate was evaporated and the residual orange oil dissolved in $50 \mathrm{ml}$ of diethyl ether. This solution was maintained at $278 \mathrm{~K}$ for at least one week, whereupon a white crystalline material deposited. The solid was filtered off, then recrystallized in ethanol to give colourless rod-like crystals of the title compound: Yield $7.87 \mathrm{~g}$ (8\%); m.p. 401-405 K; $R_{\mathrm{F}} 0.54$ (toluene/light petroleum, 10/1 v/v). Analysis for $\mathrm{C}_{8} \mathrm{H}_{8} \mathrm{Br}_{4} \mathrm{~N}_{2}$ $\left(M_{r}=451.78 \mathrm{~g} / \mathrm{mol}\right)$; Calculated (\%): C 21.27; H 1.79; N 6.20. Found (\%): C 21.41; H 1.72; N 6.10. Spectroscopic data: ${ }^{1} \mathrm{H}-$ $\mathrm{RMN}\left(\mathrm{CDCl}_{3}, 400 \mathrm{MHz}\right): \delta=4.69\left(s, 8 \mathrm{H}, \mathrm{Pz}_{-} \mathrm{CH}_{2}-\mathrm{S}\right)$ p.p.m.; ${ }^{13} \mathrm{C}-\mathrm{RMN}\left(\mathrm{CDCl}_{3}, 100 \mathrm{MHz}\right): \delta=150.41,28.75$ p.p.m. MS (EI, $70 \mathrm{eV}), \mathrm{m} / \mathrm{z}$ (\%): 452 ([ $\left.\left.\mathrm{M}^{+}\right], 11.9\right), 371$ (100), 292 (13.2), 211 (20.7), 131 (32.7), 92 (20.4), 65 (18.8); IR ( $\mathrm{KBr}$ disc, $\mathrm{cm}^{-1}$ ): $3030 w, 2977 w, 1438 s, 1405 s, 1220 s, 1096$ m, $923 w, 787 s$, $731 m, 629 m, 596 w, 543 m, 445 m$.

\section{Refinement}

Crystal data, data collection and structure refinement details are summarized in Table 3. The $\mathrm{C}$-bound $\mathrm{H}$ atoms were included in calculated positions and treated as riding atoms: $\mathrm{C}-\mathrm{H}=0.97 \AA$ with $U_{\text {iso }}(\mathrm{H})=1.2 U_{\text {eq }}(\mathrm{C})$.

\section{Acknowledgements}

This work was supported by the Swiss National Science Foundation and the University of Neuchâtel.

\section{References}

Allen, F. H. (2002). Acta Cryst. B58, 380-388.

Assoumatine, T. (1999). PhD thesis, University of Neuchâtel, Switzerland.

Assoumatine, T., Gasser, G. \& Stoeckli-Evans, H. (2007). Acta Cryst. C63, o219-o222.

Ferigo, M., Bonhote, P., Marty, W. \& Stoeckli-Evans, H. (1994). J. Chem. Soc. Dalton Trans. pp. 1549-1554.

Gasser, G. \& Stoeckli-Evans, H. (2007). Private communication (refcode ADUXAZ). CCDC, Cambridge, England.

Macrae, C. F., Bruno, I. J., Chisholm, J. A., Edgington, P. R., McCabe, P., Pidcock, E., Rodriguez-Monge, L., Taylor, R., van de Streek, J. \& Wood, P. A. (2008). J. Appl. Cryst. 41, 466-470.

Pacifico, J. \& Stoeckli-Evans, H. (2004). Acta Cryst. C60, o152-o155.

Parsons, S. \& Flack, H. (2004). Acta Cryst. A60, s61.

Sheldrick, G. M. (2008). Acta Cryst. A64, 112-122.

Spek, A. L. (2009). Acta Cryst. D65, 148-155.

Stoe \& Cie (2004). IPDSI Bedienungshandbuch. Stoe \& Cie GmbH, Darmstadt, Germany.

Westrip, S. P. (2010). J. Appl. Cryst. 43, 920-925. 


\section{supporting information}

Acta Cryst. (2014). E70, 51-53 [doi:10.1107/S1600536814011337]

\section{Crystal structure of a tetrakis-substituted pyrazine compound: 2,3,5,6-tetrakis- (bromomethyl)pyrazine}

\section{Tokouré Assoumatine and Helen Stoeckli-Evans}

\section{Computing details}

Data collection: EXPOSE in IPDS-I (Stoe \& Cie, 2004); cell refinement: CELL in IPDS-I (Stoe \& Cie, 2004); data reduction: INTEGRATE in IPDS-I (Stoe \& Cie, 2004); program(s) used to solve structure: SHELXS97 (Sheldrick, 2008); program(s) used to refine structure: SHELXL2013 (Sheldrick, 2008); molecular graphics: Mercury (Macrae et al., 2008); software used to prepare material for publication: SHELXL2013 (Sheldrick, 2008), PLATON (Spek, 2009) and publCIF (Westrip, 2010).

\section{2,3,5,6-Tetrakis(bromomethyl)pyrazine}

\section{Crystal data}

$\mathrm{C}_{8} \mathrm{H}_{8} \mathrm{Br}_{4} \mathrm{~N}_{2}$ $M_{r}=451.80$

Tetragonal, $P 4_{1} 22_{1}$

Hall symbol: P 4abw 2nw

$a=9.6858(4) \AA$

$c=26.5116(17) \AA$

$V=2487.2(3) \AA^{3}$

$Z=8$

$F(000)=1680$

\section{Data collection}

Stoe IPDS 1 diffractometer

Radiation source: fine-focus sealed tube Plane graphite monochromator $\varphi$ rotation scans Absorption correction: multi-scan

(MULscanABS in PLATON; Spek, 2009)

$T_{\min }=0.430, T_{\max }=1.000$

Refinement

Refinement on $F^{2}$

Least-squares matrix: full

$R\left[F^{2}>2 \sigma\left(F^{2}\right)\right]=0.043$

$w R\left(F^{2}\right)=0.096$

$S=0.84$

2417 reflections

127 parameters

0 restraints
$D_{\mathrm{x}}=2.413 \mathrm{Mg} \mathrm{m}^{-3}$

Mo $K \alpha$ radiation, $\lambda=0.71073 \AA$

Cell parameters from 5000 reflections

$\theta=2.2-26.0^{\circ}$

$\mu=12.91 \mathrm{~mm}^{-1}$

$T=293 \mathrm{~K}$

Rod, colourless

$0.50 \times 0.40 \times 0.30 \mathrm{~mm}$

19463 measured reflections

2417 independent reflections

1276 reflections with $I>2 \sigma(I)$

$R_{\text {int }}=0.113$

$\theta_{\text {max }}=26.0^{\circ}, \theta_{\min }=2.2^{\circ}$

$h=-11 \rightarrow 11$

$k=-11 \rightarrow 11$

$l=-32 \rightarrow 32$

Primary atom site location: structure-invariant direct methods

Secondary atom site location: difference Fourier map

Hydrogen site location: inferred from neighbouring sites

$\mathrm{H}$-atom parameters constrained

$w=1 /\left[\sigma^{2}\left(F_{0}^{2}\right)+(0.0401 P)^{2}\right]$

where $P=\left(F_{\mathrm{o}}^{2}+2 F_{\mathrm{c}}^{2}\right) / 3$ 
$(\Delta / \sigma)_{\max }<0.001$

$\Delta \rho_{\max }=0.69 \mathrm{e} \AA^{-3}$

$\Delta \rho_{\min }=-0.54$ e $\AA^{-3}$
Absolute structure: Flack $x$ determined using 419 quotients $\left[\left(\mathrm{I}^{+}\right)-\left(\mathrm{I}^{-}\right)\right] /\left[\left(\mathrm{I}^{+}\right)+\left(\mathrm{I}^{-}\right)\right]$(Parsons \& Flack, 2004)

Absolute structure parameter: $0.04(4)$

Special details

Geometry. All e.s.d.'s (except the e.s.d. in the dihedral angle between two l.s. planes) are estimated using the full covariance matrix. The cell e.s.d.'s are taken into account individually in the estimation of e.s.d.'s in distances, angles and torsion angles; correlations between e.s.d.'s in cell parameters are only used when they are defined by crystal symmetry. An approximate (isotropic) treatment of cell e.s.d.'s is used for estimating e.s.d.'s involving 1.s. planes.

Fractional atomic coordinates and isotropic or equivalent isotropic displacement parameters $\left(\AA^{2}\right)$

\begin{tabular}{lllll}
\hline & $x$ & $y$ & $z$ & $U_{\text {iso }} / U_{\text {eq }}$ \\
\hline Br1 & $1.2259(2)$ & $0.2336(2)$ & $0.31902(9)$ & $0.0928(7)$ \\
Br2 & $0.97546(18)$ & $0.47643(17)$ & $0.18421(6)$ & $0.0654(5)$ \\
N1 & $0.8843(11)$ & $0.1271(10)$ & $0.3022(5)$ & $0.043(3)$ \\
C1 & $1.0492(15)$ & $0.3062(16)$ & $0.3026(6)$ & $0.058(4)$ \\
H1A & 1.0016 & 0.3313 & 0.3335 & $0.069^{*}$ \\
H1B & 1.0608 & 0.3893 & 0.2827 & $0.069^{*}$ \\
C2 & $0.9639(14)$ & $0.2070(12)$ & $0.2742(5)$ & $0.045(3)$ \\
C3 & $0.9590(13)$ & $0.2017(12)$ & $0.2214(4)$ & $0.038(3)$ \\
C4 & $1.0501(16)$ & $0.2865(14)$ & $0.1876(5)$ & $0.053(4)$ \\
H4A & 1.0525 & 0.2463 & 0.1541 & $0.063^{*}$ \\
H4B & 1.1435 & 0.2883 & 0.2008 & $0.063^{*}$ \\
Br3 & $0.02090(19)$ & $-0.23131(16)$ & $0.06101(6)$ & $0.0663(5)$ \\
Br4 & $0.2379(2)$ & $0.4746(2)$ & $0.07000(7)$ & $0.0854(6)$ \\
N2 & $0.1293(10)$ & $0.1274(11)$ & $0.0529(5)$ & $0.042(4)$ \\
C5 & $0.3130(15)$ & $0.2938(15)$ & $0.0568(6)$ & $0.060(4)$ \\
H5A & 0.3995 & 0.3030 & 0.0387 & $0.072^{*}$ \\
H5B & 0.3317 & 0.2476 & 0.0885 & $0.072^{*}$ \\
C6 & $0.2145(13)$ & $0.2082(13)$ & $0.0262(4)$ & $0.041(3)$ \\
C7 & $0.0442(13)$ & $0.0470(13)$ & $0.0261(4)$ & $0.039(3)$ \\
C8 & $-0.0509(16)$ & $-0.0420(17)$ & $0.0578(5)$ & $0.056(4)$ \\
H8A & -0.1426 & -0.0425 & 0.0432 & $0.068^{*}$ \\
H8B & -0.0575 & -0.0041 & 0.0916 & $0.068^{*}$ \\
& & & &
\end{tabular}

Atomic displacement parameters $\left(\AA^{2}\right)$

\begin{tabular}{lllllll}
\hline & $U^{11}$ & $U^{22}$ & $U^{33}$ & $U^{12}$ & $U^{13}$ & $U^{23}$ \\
\hline $\mathrm{Br} 1$ & $0.0587(12)$ & $0.0884(15)$ & $0.1313(15)$ & $0.0040(9)$ & $-0.0371(11)$ & $-0.0122(14)$ \\
$\mathrm{Br} 2$ & $0.0729(11)$ & $0.0512(9)$ & $0.0719(10)$ & $0.0017(7)$ & $0.0043(10)$ & $0.0119(9)$ \\
$\mathrm{N} 1$ & $0.046(9)$ & $0.046(9)$ & $0.037(7)$ & $0.002(5)$ & $-0.002(5)$ & $0.002(5)$ \\
$\mathrm{C} 1$ & $0.056(9)$ & $0.059(9)$ & $0.058(9)$ & $-0.009(8)$ & $-0.009(8)$ & $-0.011(8)$ \\
$\mathrm{C} 2$ & $0.051(8)$ & $0.035(7)$ & $0.050(7)$ & $0.004(6)$ & $-0.003(7)$ & $-0.008(6)$ \\
$\mathrm{C} 3$ & $0.034(7)$ & $0.037(7)$ & $0.043(7)$ & $0.003(6)$ & $0.001(6)$ & $-0.003(6)$ \\
$\mathrm{C} 4$ & $0.053(9)$ & $0.047(8)$ & $0.059(8)$ & $0.004(6)$ & $0.007(8)$ & $0.002(7)$ \\
$\mathrm{Br} 3$ & $0.0813(12)$ & $0.0468(9)$ & $0.0710(9)$ & $-0.0060(8)$ & $-0.0011(10)$ & $0.0101(8)$ \\
$\mathrm{Br} 4$ & $0.0862(13)$ & $0.0616(11)$ & $0.1086(15)$ & $-0.0065(9)$ & $0.0033(12)$ & $-0.0330(11)$
\end{tabular}


supporting information

\begin{tabular}{lllllll}
$\mathrm{N} 2$ & $0.046(9)$ & $0.050(9)$ & $0.030(6)$ & $-0.006(5)$ & $-0.007(5)$ & $0.003(5)$ \\
$\mathrm{C} 5$ & $0.053(9)$ & $0.073(10)$ & $0.054(9)$ & $-0.009(8)$ & $-0.015(8)$ & $-0.006(8)$ \\
C6 & $0.047(8)$ & $0.043(7)$ & $0.033(6)$ & $0.007(6)$ & $-0.002(6)$ & $-0.002(6)$ \\
C7 & $0.036(7)$ & $0.040(7)$ & $0.042(6)$ & $-0.003(6)$ & $0.000(6)$ & $0.005(6)$ \\
C8 & $0.060(9)$ & $0.062(9)$ & $0.047(7)$ & $0.001(8)$ & $0.000(8)$ & $0.007(8)$ \\
\hline
\end{tabular}

Geometric parameters $\left(\AA,{ }^{\circ}\right)$

\begin{tabular}{|c|c|c|c|}
\hline $\mathrm{Br} 1-\mathrm{C} 1$ & $1.901(15)$ & $\mathrm{Br} 3-\mathrm{C} 8$ & $1.963(17)$ \\
\hline $\mathrm{Br} 2-\mathrm{C} 4$ & $1.978(14)$ & $\mathrm{Br} 4-\mathrm{C} 5$ & $1.928(15)$ \\
\hline $\mathrm{N} 1-\mathrm{C} 2$ & $1.319(17)$ & $\mathrm{N} 2-\mathrm{C} 7$ & $1.337(15)$ \\
\hline $\mathrm{N} 1-\mathrm{C} 3^{\mathrm{i}}$ & $1.334(15)$ & $\mathrm{N} 2-\mathrm{C} 6$ & $1.339(16)$ \\
\hline $\mathrm{C} 1-\mathrm{C} 2$ & $1.474(18)$ & $\mathrm{C} 5-\mathrm{C} 6$ & $1.502(17)$ \\
\hline $\mathrm{C} 1-\mathrm{H} 1 \mathrm{~A}$ & 0.9700 & $\mathrm{C} 5-\mathrm{H} 5 \mathrm{~A}$ & 0.9700 \\
\hline $\mathrm{C} 1-\mathrm{H} 1 \mathrm{~B}$ & 0.9700 & C5-H5B & 0.9700 \\
\hline $\mathrm{C} 2-\mathrm{C} 3$ & $1.402(15)$ & $\mathrm{C} 6-\mathrm{C6}^{\mathrm{ii}}$ & $1.39(2)$ \\
\hline $\mathrm{C} 3-\mathrm{N} 1^{\mathrm{i}}$ & $1.334(15)$ & $\mathrm{C} 7-\mathrm{C}^{\mathrm{ii}}$ & $1.39(2)$ \\
\hline $\mathrm{C} 3-\mathrm{C} 4$ & $1.504(17)$ & $\mathrm{C} 7-\mathrm{C} 8$ & $1.516(17)$ \\
\hline $\mathrm{C} 4-\mathrm{H} 4 \mathrm{~A}$ & 0.9700 & $\mathrm{C} 8-\mathrm{H} 8 \mathrm{~A}$ & 0.9700 \\
\hline $\mathrm{C} 4-\mathrm{H} 4 \mathrm{~B}$ & 0.9700 & $\mathrm{C} 8-\mathrm{H} 8 \mathrm{~B}$ & 0.9700 \\
\hline $\mathrm{C} 2-\mathrm{N} 1-\mathrm{C} 3^{\mathrm{i}}$ & $117.9(13)$ & $\mathrm{C} 7-\mathrm{N} 2-\mathrm{C} 6$ & $116.1(12)$ \\
\hline $\mathrm{C} 2-\mathrm{C} 1-\mathrm{Br} 1$ & $112.4(10)$ & $\mathrm{C} 6-\mathrm{C} 5-\mathrm{Br} 4$ & $111.1(9)$ \\
\hline $\mathrm{C} 2-\mathrm{C} 1-\mathrm{H} 1 \mathrm{~A}$ & 109.1 & $\mathrm{C} 6-\mathrm{C} 5-\mathrm{H} 5 \mathrm{~A}$ & 109.4 \\
\hline $\mathrm{Br} 1-\mathrm{C} 1-\mathrm{H} 1 \mathrm{~A}$ & 109.1 & $\mathrm{Br} 4-\mathrm{C} 5-\mathrm{H} 5 \mathrm{~A}$ & 109.4 \\
\hline $\mathrm{C} 2-\mathrm{C} 1-\mathrm{H} 1 \mathrm{~B}$ & 109.1 & $\mathrm{C} 6-\mathrm{C} 5-\mathrm{H} 5 \mathrm{~B}$ & 109.4 \\
\hline $\mathrm{Br} 1-\mathrm{C} 1-\mathrm{H} 1 \mathrm{~B}$ & 109.1 & $\mathrm{Br} 4-\mathrm{C} 5-\mathrm{H} 5 \mathrm{~B}$ & 109.4 \\
\hline $\mathrm{H} 1 \mathrm{~A}-\mathrm{C} 1-\mathrm{H} 1 \mathrm{~B}$ & 107.9 & $\mathrm{H} 5 \mathrm{~A}-\mathrm{C} 5-\mathrm{H} 5 \mathrm{~B}$ & 108.0 \\
\hline $\mathrm{N} 1-\mathrm{C} 2-\mathrm{C} 3$ & $121.3(11)$ & $\mathrm{N} 2-\mathrm{C} 6-\mathrm{C}^{\mathrm{ii}}$ & $121.7(7)$ \\
\hline $\mathrm{N} 1-\mathrm{C} 2-\mathrm{C} 1$ & $115.0(12)$ & $\mathrm{N} 2-\mathrm{C} 6-\mathrm{C} 5$ & $115.4(11)$ \\
\hline $\mathrm{C} 3-\mathrm{C} 2-\mathrm{C} 1$ & $123.6(12)$ & $\mathrm{C} 6{ }^{\mathrm{ii}}-\mathrm{C} 6-\mathrm{C} 5$ & $122.9(8)$ \\
\hline $\mathrm{N} 1-\mathrm{C} 3-\mathrm{C} 2$ & $120.8(11)$ & $\mathrm{N} 2-\mathrm{C} 7-\mathrm{C}^{\mathrm{ii}}$ & $121.9(7)$ \\
\hline $\mathrm{N} 1-\mathrm{C} 3-\mathrm{C} 4$ & $115.3(12)$ & $\mathrm{N} 2-\mathrm{C} 7-\mathrm{C} 8$ & $114.3(11)$ \\
\hline $\mathrm{C} 2-\mathrm{C} 3-\mathrm{C} 4$ & $123.8(11)$ & $\mathrm{C} 7^{\mathrm{ii}}-\mathrm{C} 7-\mathrm{C} 8$ & $123.7(7)$ \\
\hline $\mathrm{C} 3-\mathrm{C} 4-\mathrm{Br} 2$ & $108.7(9)$ & $\mathrm{C} 7-\mathrm{C} 8-\mathrm{Br} 3$ & $109.9(10)$ \\
\hline $\mathrm{C} 3-\mathrm{C} 4-\mathrm{H} 4 \mathrm{~A}$ & 109.9 & $\mathrm{C} 7-\mathrm{C} 8-\mathrm{H} 8 \mathrm{~A}$ & 109.7 \\
\hline $\mathrm{Br} 2-\mathrm{C} 4-\mathrm{H} 4 \mathrm{~A}$ & 109.9 & $\mathrm{Br} 3-\mathrm{C} 8-\mathrm{H} 8 \mathrm{~A}$ & 109.7 \\
\hline $\mathrm{C} 3-\mathrm{C} 4-\mathrm{H} 4 \mathrm{~B}$ & 109.9 & $\mathrm{C} 7-\mathrm{C} 8-\mathrm{H} 8 \mathrm{~B}$ & 109.7 \\
\hline $\mathrm{Br} 2-\mathrm{C} 4-\mathrm{H} 4 \mathrm{~B}$ & 109.9 & $\mathrm{Br} 3-\mathrm{C} 8-\mathrm{H} 8 \mathrm{~B}$ & 109.7 \\
\hline $\mathrm{H} 4 \mathrm{~A}-\mathrm{C} 4-\mathrm{H} 4 \mathrm{~B}$ & 108.3 & $\mathrm{H} 8 \mathrm{~A}-\mathrm{C} 8-\mathrm{H} 8 \mathrm{~B}$ & 108.2 \\
\hline $\mathrm{C} 3-\mathrm{N} 1-\mathrm{C} 2-\mathrm{C} 3$ & $-0.2(16)$ & $\mathrm{C} 2-\mathrm{C} 3-\mathrm{C} 4-\mathrm{Br} 2$ & $-78.6(15)$ \\
\hline $\mathrm{C} 3-\mathrm{i} 1-\mathrm{C} 2-\mathrm{C} 1$ & $175.9(12)$ & $\mathrm{C} 7-\mathrm{N} 2-\mathrm{C} 6-\mathrm{C}^{\mathrm{ii}}$ & $4(2)$ \\
\hline $\mathrm{Br} 1-\mathrm{C} 1-\mathrm{C} 2-\mathrm{N} 1$ & $91.3(13)$ & $\mathrm{C} 7-\mathrm{N} 2-\mathrm{C} 6-\mathrm{C} 5$ & $-177.8(12)$ \\
\hline $\mathrm{Br} 1-\mathrm{C} 1-\mathrm{C} 2-\mathrm{C} 3$ & $-92.6(15)$ & $\mathrm{Br} 4-\mathrm{C} 5-\mathrm{C} 6-\mathrm{N} 2$ & $-93.3(12)$ \\
\hline $\mathrm{N} 1-\mathrm{C} 2-\mathrm{C} 3-\mathrm{N} 1^{\mathrm{i}}$ & $0.3(19)$ & $\mathrm{Br} 4-\mathrm{C} 5-\mathrm{C} 6-\mathrm{C}^{\mathrm{ii}}$ & $84.8(17)$ \\
\hline $\mathrm{C} 1-\mathrm{C} 2-\mathrm{C} 3-\mathrm{N} 1^{\mathrm{i}}$ & $-175.5(12)$ & $\mathrm{C} 6-\mathrm{N} 2-\mathrm{C} 7-\mathrm{C}^{\mathrm{ii}}$ & $2(2)$ \\
\hline $\mathrm{N} 1-\mathrm{C} 2-\mathrm{C} 3-\mathrm{C} 4$ & $-177.9(12)$ & $\mathrm{C} 6-\mathrm{N} 2-\mathrm{C} 7-\mathrm{C} 8$ & $-179.7(12)$ \\
\hline
\end{tabular}




\section{supporting information}

$\begin{array}{llll}\mathrm{C} 1-\mathrm{C} 2-\mathrm{C} 3-\mathrm{C} 4 & 6(2) & \mathrm{N} 2-\mathrm{C} 7-\mathrm{C} 8-\mathrm{Br} 3 & -101.0(12) \\ \mathrm{N} 1-\mathrm{C} 3-\mathrm{C} 4-\mathrm{Br} 2 & 103.1(11) & \mathrm{C} 7 \mathrm{ii}-\mathrm{C} 7-\mathrm{C} 8-\mathrm{Br} 3 & 77.4(18)\end{array}$

Symmetry codes: (i) $-y+1,-x+1,-z+1 / 2$; (ii) $y, x,-z$.

Hydrogen-bond geometry $\left(A,{ }^{\circ}\right)$

\begin{tabular}{lllll}
\hline$D-\mathrm{H} \cdots A$ & $D-\mathrm{H}$ & $\mathrm{H} \cdots A$ & $D \cdots A$ & $D-\mathrm{H} \cdots A$ \\
\hline $\mathrm{C} 1-\mathrm{H} 1 A \cdots \mathrm{Br} 2^{\mathrm{iii}}$ & 0.97 & 3.02 & $3.863(14)$ & 146 \\
$\mathrm{C} 1-\mathrm{H} 1 B \cdots \mathrm{Br} 2$ & 0.97 & 2.86 & $3.617(16)$ & 135 \\
$\mathrm{C} 5-\mathrm{H} 5 A \cdots \mathrm{Br} 4^{\mathrm{ii}}$ & 0.97 & 3.04 & $3.748(16)$ & 131 \\
$\mathrm{C} 5-\mathrm{H} 5 B \cdots \mathrm{Br} 3^{\mathrm{iv}}$ & 0.97 & 3.03 & $3.864(14)$ & 145 \\
$\mathrm{C} 8-\mathrm{H} 8 A \cdots \mathrm{Br} 3^{\mathrm{ii}}$ & 0.97 & 2.96 & $3.654(15)$ & 130 \\
\hline
\end{tabular}

Symmetry codes: (ii) $y, x,-z$; (iii) $-y+3 / 2, x-1 / 2, z+1 / 4$; (iv) $-x+1 / 2, y+1 / 2,-z+1 / 4$. 